\title{
Transforming a research paper into a rich internet publication
}

\author{
Leen Breure * \\ Utrecht University, Utrecht, The Netherlands \\ and \\ DANS (Data Archiving and Networked Services), The Hague, The Netherlands
}

\begin{abstract}
Scientific communication is gradually changing. In the web era the genre of the printed paper has survived in digital format as static PDF, but annotations, multimedia and access to data are appearing as new interactive features of innovative scholarly journals. Together they justify the term Rich Internet Publication (RIP) as name for a new emerging genre. Because of the authoritative role of publications in highly ranked journals, a RIP is mostly used as a 'showcase' to publish supplementary material that could not be made available otherwise, and to generate a broader interest in a project. The traditional authoring of linear text does not adequately fit the new situation, in which the author has to deal with much more information in various formats and has access to new options for presentation. This paper describes a strategy to transform a regular paper into a RIP. The strategy is divided into a conceptual stage, in which focal points are created and a balanced mixture of different media are achieved divided over different levels, and the implementation.
\end{abstract}

Keywords: Multimedia, interactivity, visualization, data supplement, modeling, web technology, enhanced publication

\section{Introduction}

This paper describes a procedure for converting a regular scholarly publication into an interactive multimedia publication, also known as a Rich Internet Publication (RIP). We have defined the concept RIP elsewhere [5] and have used it as an overarching term, which covers a broad range of digital publications enhanced with visuals, interactivity and research data. At the low end it is text-based, in its most simple form a digital bundle of an article with annexes (also known as an 'enhanced publication' $[13,15]$ ), more advanced an enriched text with different levels of detail and interactive components, and at the high-end it comes close to a real web application. ${ }^{1}$

Hyperlinking can be used as mechanism to connect an article with annexes, which results in a RIP as a package of documents and data. The enhanced publication, introduced some years ago, is based on this model (see Fig. 1). A drawback of this package concept is that it makes the reader hop from one document to another; data is linked as a file, which has to be downloaded first and then opened offline in an appropriate application. The current state of online publishing has brought more sophisticated solutions within the reach of authors that have basic knowledge of web authoring. A more advanced RIP has no longer a linear structure, but is a tree or network of sections, each combining text with

\footnotetext{
*Address for correspondence: Leen Breure, DANS, Postbox 93067, 2509 AB, The Hague, The Netherlands. E-mail: L.Breure@uu.nl.

${ }^{1}$ The concept Rich Internet Publication should not be confused with a Rich Internet Application (RIA): the first one is predominantly text-based, while the latter is component-based and algorithm-driven. For a definition of a RIA, see for example $[12]$.
} 


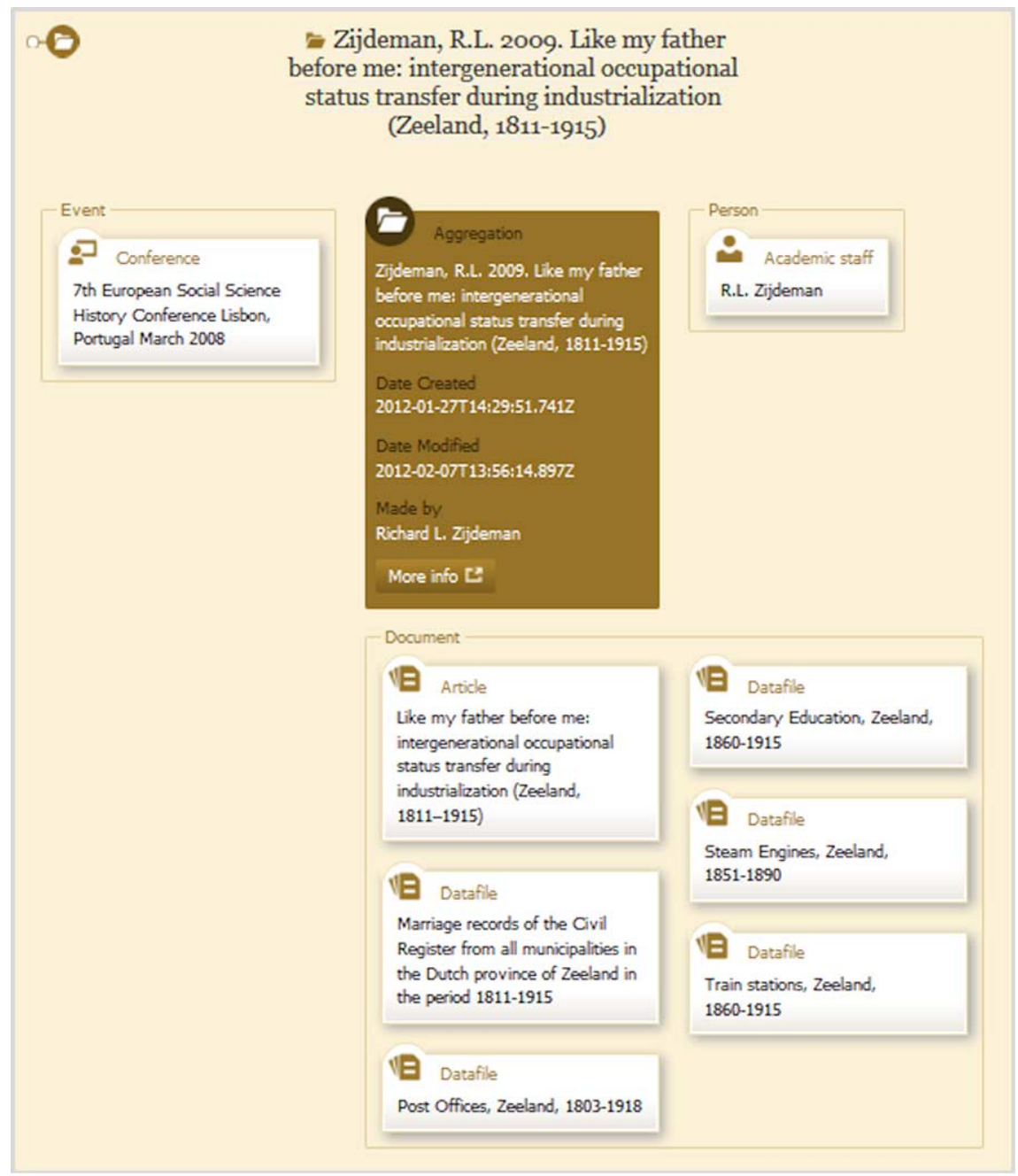

Fig. 1. Visualization of the structure of an enhanced publication (simple RIP). An article is connected with a dataset and information about the conference and author. ${ }^{2}$ (Colors are visible in the online version of the article; http://dx.doi.org/10.3233/ ISU-140757.)

multimedia and data. Depending on the technology available an interactive view of the research data can be embedded in the text through data widgets, which creates a more integrated presentation.

A RIP is in a sense an ideal type. You may not easily find many examples that have all features mentioned, ${ }^{3}$ but a "RIP trend" is clearly visible in the innovation in scholarly products of major publishers (in journals, but also e-books and apps). Elsevier's Article of the Future has been trend setting in this respect [1]. A RIP may also appear outside a journal, published on an institutional or private website, as a more elaborate stand-alone version of a regular research paper. There are specific reasons for creating such a publication:

\footnotetext{
${ }^{2}$ Source: http://www.narcis.nl/vpub/RecordID/escape-demo\%3Arem\%3A2679/id/2/Language/EN.

${ }^{3} \mathrm{An}$ overview of features is to be found at: http://xposre.nl/epfeatures/index.html.
} 
1. Journals, even those who are fully online, set limits on the acceptance of multimedia content and other additional material.

2. A RIP opens the possibility to interactively exploring the research data on which a paper is based.

3. Use of multimedia and access to the underlying data make a RIP an attractive showcase that may generate a broader interest in the project.

The larger amount and greater complexity of information has consequences for the structure of the publication. Simply expanding a text will easily cause information overload, which is to be prevented by some form of interactive selectivity. This can be achieved by a concise top layer that arouses interest and which is linked to a more extended detail level (which, for example, may be followed by a next level of documentation), in this way allowing the user to actively explore the content and to select information according to his/her current interest. Darnton proposed such a pyramid structure for e-books [10].

The major hurdle in transforming a paper into the direction of the novel genre is letting go the familiar model of the printed publication, which has successfully survived in the digital era (both in HTML and PDF format) and in which we all have been trained so well. Its linear character makes authoring a stepby-step process, while there are many more options with non-linear variants. Several problems present themselves to authors: how to find an appropriate (multilevel) structure, which topics should get focus, what should be visualized and what described, how to get the most out of multimedia (in other words: how to "write" with images and other non-textual media). To get out of this confusion a relatively simple scheme as outlined in this paper may be helpful; it is based on more than five year experience in creating RIPs, both in Flash and HTML/Javascript, but it is not a recipe that can be followed blindly. In all stages some discernment and feeling for multimedia and presentation are required to take appropriate decisions. We shall focus on interactive multimedia web documents with a highly visual profile but still containing a lot of text, rather than on the package variant. Since publication structure and content greatly vary by scientific field, the following will be most in line with domains that largely rely on narrative text like the humanities and the social sciences.

\section{Experiments in authoring and modeling}

In a series of projects we have done action research and have experimented with authoring methods and informal modeling techniques in order to establish an approach for this complex authoring process. In an early stage work comprised Flash applications, particularly concerning cultural landscapes [2, 4], which was extended to the field of cultural heritage in general. ${ }^{4}$ Subjects varied from local castles, peat lakes and wetlands to medieval memorial culture. In all projects content experts (i.e. scholars) collaborated closely with technical staff, in feed-back sessions on weekly basis. In these sessions the team not only developed the storyline from top to bottom and evaluated the digital product as far as it was ready, but also discussed and learned about the various forms of presentation. The first applications were completely handcrafted. In the next phase authoring tools have been developed (both Flash- and HTMLbased) that incorporate our earlier experience and many of the principles of enhanced publications that have been implemented elsewhere in journals and demos. This Xpos're software ${ }^{5}$ allow scholars with basic web authoring skills (and/or some technical assistance) to create and publish a multimedia article in XML format [3]. Currently, the technical scope is extended to fully responsive HTML5 templates with embedded Javascript code for client-side formatting on display.

\footnotetext{
${ }^{4}$ See portfolio on http://xposre.nl/.

${ }^{5}$ See http://xposre.nl/software/index.html.
} 
Prior to the implementation a relatively technology independent specification of the structure and the presentation mechanisms of the publication is required, the so-called conceptual model. It allows the author to concentrate on 'what' and 'how' without having to worry about details of implementation. However, in practice, one will keep the target platform in mind for practical reasons: certain widgets like zoomable images, charts and slideshows may be standard in one environment and much more difficult to obtain for others. In the next stage, the implementation, the conceptual model is matched with the features, available components and limitations of the publication system.

\section{Conceptual modeling}

\subsection{Preliminaries}

In our experience authors like to start from something familiar such as a conventional paper, which is then transformed into a RIP. The purpose of a conceptual modeling strategy is to make this transformation a systematic activity. It helps to organize the (large amount of) content in such a way that the attention of the user is effectively drawn to the main points of the message that is to be conveyed and information is quickly and easily understood through effective use of the new media.

There are two related modeling domains, which should be mentioned here for the sake of completeness. Hypermedia modeling, in particular of adaptable hypermedia documents, has dealt with the variety in a user population and introduced the notion of a hierarchical hyperspace of interlinked pages [6,14]. In addition, there is a rather extensive but in this context less relevant literature on modeling Rich Internet Applications [7], which, as the name suggests, are more real programs rather than interactive multimedia texts (see also note 1).

As a starting point we assume, that:

A. there is a narrative text in the form of a (published) paper or at least a rough version of it, as usually structured into sections (see Fig. 2), and

B. that the publication's subject has some relevant visual aspects or can be related to material objects. It may be difficult to convert a publication on a completely abstract subject into a multimedia RIP.

\subsection{Step 1 - Creating focus}

As noted above, a large amount of information can be divided into different levels of increasing detail. The top level is the first one read by the user; here attention should be drawn to the main points. Therefore, the first step is selection of significant passages in the text that are essential to the line of discourse. This is similar to underlining or highlighting text fragments when you study a narrative text in depth. They should cover the same aspects that are included in a good abstract: background and context,
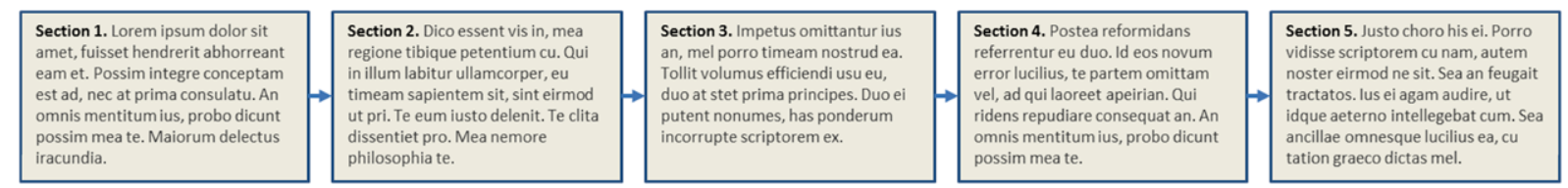

Fig. 2. Starting point: a linear text divided into sections. (Colors are visible in the online version of the article; http://dx.doi.org/ 10.3233/ISU-140757.) 

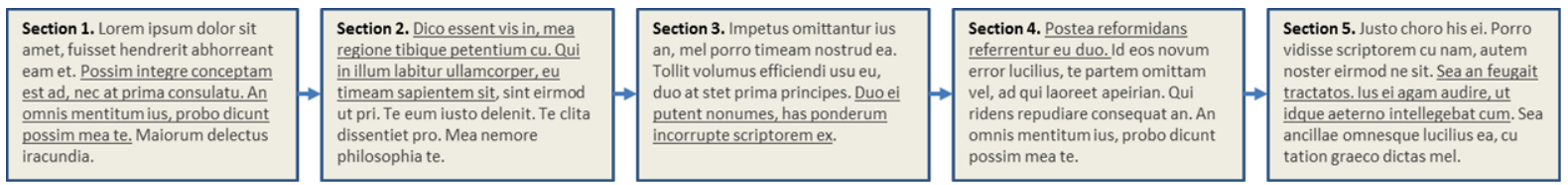

Fig. 3. Step 1 - Focus created in the linear text: core passages selected (in a real paper, of course, much longer than in this diagram). (Colors are visible in the online version of the article; http://dx.doi.org/10.3233/ISU-140757.)

research problem, object of study, aims and objectives, methods, results and conclusion. This divides the narrative into two sets of text fragments: core passages assigned to the top level and a next level of the associated elaborations (see Fig. 3). We shall come back to the overall structure in step 3.

An important consideration are the entry points. If we may assume that the RIP is read from the beginning to the end, the first core passage is the single entry point. However, since a hypertext network can be traversed in many directions, one may also choose to have more than one entry point at the top. This presupposes that nodes to some extent can be individually understood without the necessary knowledge of previous content.

\subsection{Step 2 - Visualizing focus}

Next we should check where text is the optimal medium and where it is not. Because of our familiarity with descriptions we may easily forget that we have got the means to display what is visible, to show movement and developments, and to make the user an active participant in knowledge gathering, rather than an passive consumer. Active in this context also implies some freedom in choosing a path through the network of hyperlinked content.

Visualization is the hardest part of making a RIP that is also 'rich' in terms of the media used. In practice many people seem to have difficulty with finding the right visuals if a direct image of an object or person is not applicable. Thinking of the material aspects of a topic proves to be helpful: What parts can be seen or heard? Are there any concrete things related to it? A great variety of possible relationships exists between a visual and the associated subject:

- a visual may represent an example of the phenomenon discussed,

- it may be an icon (a pars pro toto reference, as many icons on a computer desktop),

- it may put the subject in its context (e.g. a wall chart of animals in their natural habitat),

- show structure (e.g. anatomical chart) or

- geographical position (point or area on a map) or

- represent a process (e.g. the growth of an organism).

Ideally, each core passage is to be matched with an image that in some way visualizes its message. The broader term 'visual' is more correct. The visual may be part of the research content itself (e.g. a picture of a research object), or be constructed by the researcher (a chart, diagram, map). The visual should be immediately understood: complex compositions or video's will not work. Its primary role is similar to a sign board of a shop: it identifies the nature and location of a topic within the RIP. But it can do more and act as a signpost at a node in the network, as a visual index and access point allowing the user to choose between different paths. For example, interactive graphics with hotspots are used for linking with other texts and/or data. An image with clickable spots can present a synoptic view of a topic and at the same time through hyperlinks or scripting provide access to underlying information about aspects of that topic 
(e.g. the Anatomy Explorer $\left.{ }^{6}\right)$. In the same way an interactive map has places, on which can be clicked to display information about a location.

In almost all cases the image cannot stand alone and will need at least a caption to clarify its constituent parts, otherwise most users will overlook relevant details. This caption can be merged with the current core passage and form in this way a separate text (dominated by the visual component). The core passage was part of a larger text; the remaining part, labeled above as elaboration, is then turned into a second level text linked to the core passage. Alternatively, if splitting is not a good idea, for example for technical reasons, both core and elaboration can be kept as one text, in which the first gets a prominent place by typographical means (bold, different font, text box, etc.).

At the end of this step the presentation of core passages should have been defined, either as prominent text through typography (cf. the 'lead' in news stories) if visualization is not feasible, or by means of significant visuals with explanatory text (see Fig. 4).

\subsection{Step 3 - Restructuring content}

A normal paper is a linear text, which is supposed to be read from the beginning to the end. The author has got only poor means to indicate a latent hierarchical structure by means of the numbering of sections. An article has usually two levels: the text itself and notes plus references (which, however, may be quite extensive in the humanities). A RIP, on the other hand, is a true multilevel hierarchy or a network of linked content. The vertical paths can consist of normal hyperlinks, but where appropriate a visual index may be used as well (see step 2 above). Therefore, the source text is to be checked for hidden hierarchical relations that can be made explicit (see Fig. 5). It may make sense to subordinate and
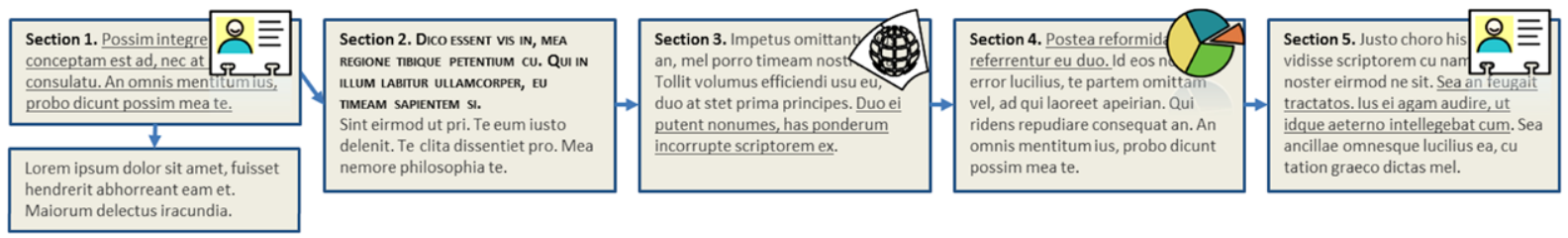

Fig. 4. Step 2 - Visual elements (image, lead, map, chart, etc.) added to the core passages: visual plus core text may become an independent text to which an elaboration is linked (left). (Colors are visible in the online version of the article; http://dx.doi.org/10.3233/ISU-140757.)
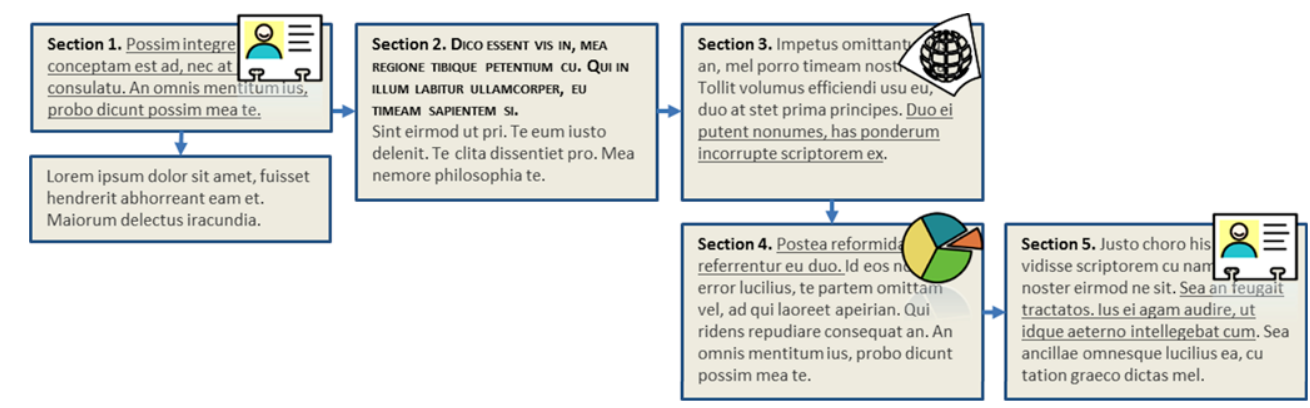

Fig. 5. Step 3 - Content restructured: implicit hierarchical structures made explicit. (Colors are visible in the online version of the article; http://dx.doi.org/10.3233/ISU-140757.)

\footnotetext{
${ }^{6}$ InnerBody: http://www.innerbody.com.
} 

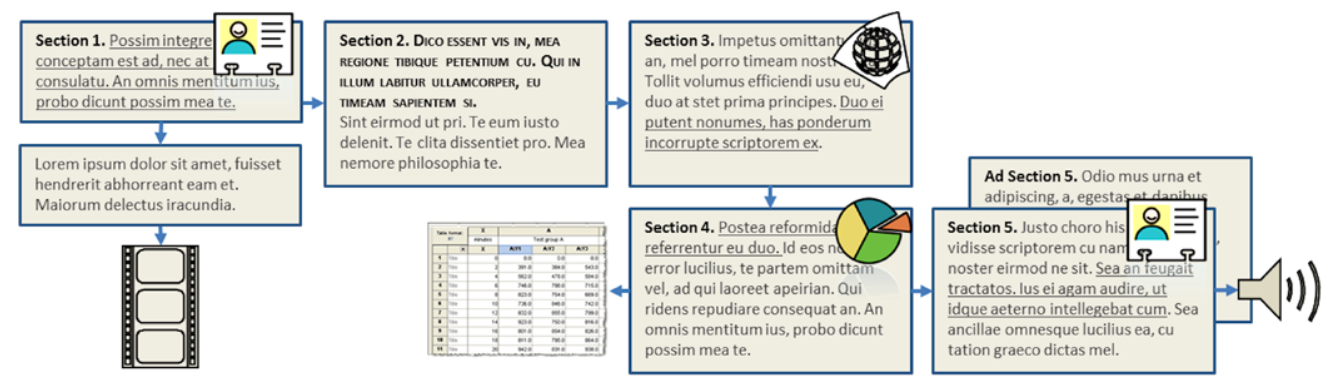

Fig. 6. Step 4 - Supplements (multimedia content or additional text) and data (or access to an online database) added. (Colors are visible in the online version of the article; http://dx.doi.org/10.3233/ISU-140757.)

link one text section to another if it is an elaboration and its reading is strictly speaking optional. Later, this can be implemented, for example, through an expandable text mechanism: the subordinated text is only displayed on mouse click. It is a good idea to keep the hierarchy shallow; deep hierarchies tend to cause usability problems in navigation.

\subsection{Step 4 - Adding supplementary information and data}

In this step one has to decide what supplementary information and data should be added and how that affects the structure of the RIP (see Fig. 6). A decisive factor to be reckoned with is the format in which this content is already available. Conceptually one can choose between the following options (combinations are possible):

1. Organize supplements in a separate level, connected through links to the main text of the RIP (i.e. to the core passages and the elaboration texts). Data can be added as an appendix as in a printed paper, or linked as a data set, but in an interactive environment access to an online spreadsheet or database may be more obvious. Embedding widgets for querying and filtering makes exploration and analysis of data possible within the context of the publication itself.

2. Insert supplementary material through multimedia objects into the main text. This method has as advantage, that a supplement is integrated with the discourse itself, but at the same time, it may distract the user's attention and encourage suspending reading. Videos are currently a rather popular medium for further explanations; animations that can be paused and include interactivity to obtain more information, are more suitable for exploratory purposes. Normal charts can be replaced with interactive versions, which display on button click the results for different experimental conditions. In a similar way interactive maps hide a lot of details, which become visible on mouse clicks ("just in time information"). A dynamic view on a spreadsheet can be embedded into a text through data widgets (for example, Figshare offers this option to preview data files).

\section{Implementation}

The conceptual modeling ends up with a multilevel set of web documents to which multimedia and supplements have been assigned. The last stage of RIP making is the implementation, which varies greatly with the type of RIP and the software platform chosen. Almost all options are ultimately traced back to a combination of HTML, CSS and Javascript, but the ways of 'packaging' content are quite 
different. Moreover, implementations differ from each other in the way the text is to be read, only online or also offline:

1. Enhanced publication as a package: In this variant, HTML pages and media files are published on a website and hold together by metadata and hyperlinks (see Fig. 1). The degree of integration from the user's point of view is rather low. It may be wise to reduce the number of physical documents because all of them are directly part of the user's navigation space, which otherwise may easily become confusing. It is most likely that the paper is more or less kept in its original form with links to supplements and multimedia objects, the creation of which is completely left to the author. For this there is a wide choice of software and tools, varying from libraries, customizable components to easy to use cloud services.

2. Article in web CMS: Another, more integrated option is a web article in a web content management system also used for blogs. If a platform like WordPress is chosen, integration of content is almost automatically enforced and navigation widgets are built-in. The choice of ready-made multimedia components that can be embedded in the text itself, is usually limited to what is available for that platform. SVG images with some scripting are excellent to implement interactive visuals [8]. Linking to external resources is possible, although this will be at the expense of the sense of integration.

3. E-book: Static e-books are frequently published as PDFs. In a PDF the well-known typographic means of a printed publication can be used such as multilevel numbering, sidebars, separate text blocks, smaller fonts etcetera to create a visual difference. Alternatively, deeper level content can be kept as separate web documents linked to the main text. Although a nice looking result can be achieved, especially if a DTP program has been used for authoring, much of the potential richness of a RIP is got lost in a PDF, at least if everything should work cross-platform (advanced interactive features of the PDF standard are not consistently supported).

An interactive, real e-book may be an interesting alternative because it can be read both online and offline. The recent EPUB3 standard allows more interactivity than EPUB2, which is still used for most e-books, but does not support scripting. An EPUB3 document is basically a zip file containing HTML5, CSS and Javascript. The new standard has already become a starting point for a niche market. An e-book can be of book length, but shorter texts also occur. Because of the prevailing linear structure of all e-books, an hierarchy that is made explicit tends to get flattened again in this format. The content of top and subordinate levels is displayed as a sequence of chapters or sections, which may be cross linked to implement hierarchical relations.

There are still some shortcomings: the popular reflowable format of EPUB3 makes that authors cannot rely on the precise position of text blocks, sidebars and other typographic elements. Besides, the current reader applications are not always reliable with regard to identical rendering of advanced features such as the alternative fixed format of EPUB3 [11]. However, thanks to the option of Javascript various widgets and interactive multimedia objects can be embedded to display secondary information. For example, in Apple iBooks footnotes can be displayed as a popup [9]. Converting an e-book to an app guarantees the best reading experience. The transformation into an app circumvents the idiosyncrasies e-book reader software.

\section{Conclusion}

Scientific publications are gradually changing. There is a growing tendency to publish a digital paper together with supplements such as images, videos, 3D-models and underlying data. More information 
requires a user-friendly structure and user interface to prevent information overload by a well-balanced presentation of main issues and matter of secondary importance. The genre of printed publications is evolving through look-alike and slightly extended digital versions to the motley collection of Rich Internet Publications. These enriched publications have different physical forms varying from PDF, web articles and web books to e-books and apps for mobile devices.

At first sight the main problem for an author may appear to be the technique, but a successful RIP requires a different way of thinking that fully embraces the non-linear character of hypermedia and treats visualization as a first class citizen. A normal (printed) publication is a safe starting point, which in a few steps can be transformed into a RIP. Publishing data in one form or another appears to be a sensitive issue, but to do this through an enhanced publication is certainly one of the safest ways, because it provides a detailed context for interpretation and secures the academic credits for authorship.

Given the current state of authoring technology many people will need some technical assistance in the implementation stage, which in itself is nothing new. Most papers are submitted as a Word file, while the final layout of the article is done by the publisher. However, in this period of transition support for the new media is not yet as well organized as it should.

A last, pressing question remains, whether it is worthwhile to do so much extra effort at all. The answer is negative if one considers only the current system of earning academic credits, but the showcase effect is not to be underestimated, also in teaching and promotion of research. The multimedia RIP supports the emerging concept of visual literacy enforced by the convergence of current web technology and the dominant role of visuals in knowledge transfer. When we look at the periphery of scholarly communication, and go to blogs, social media and popular presentations of research, where rules are few and credits come through visibility, we see already a natural link with this multimedia trend in today's society.

\section{References}

[1] I.J.J. Aalbersberg, F. Heeman, H. Koers and E. Zudilova-Seinstra, Elsevier's article of the future: enhancing the user experience and integrating data through applications, Insights: the UKSG journal 25(1) (2012), 33-43.

[2] L. Breure L and J.B. Voorbij, Visualization patterns for cultural landscapes, in: The 9th International Symposium on Virtual Reality, Archaeology and Cultural Heritage VAST (2008): Workshops, Short and Project Papers Proceedings, M. Ashley, S. Hermon, A. Proença and K. Rodriguez-Echavarria, eds, Departamento de Informatica, Universidade do Minho, Braga, pp. 91-96.

[3] L. Breure, M. Hoogerwerf and R. van Horik, Xpos're: A tool for rich internet publications, Digital Humanities Quarterly 8(2) (2014).

[4] L. Breure, W. Landman, A.G.L. Spruit and J.B. Voorbij, Modeling portals for cultural landscapes, in: Museums and the Web 2008: Selected Papers from an International Conference, J. Trant and D. Bearman, eds, Archives \& Museum Informatics, Toronto, pp. 141-150.

[5] L. Breure, H. Voorbij and M. Hoogerwerf, Rich internet publications: "Show what you tell", Journal of Digital Information, North America 12 (2011).

[6] P. Brusilovsky, Developing adaptive educational hypermedia systems: From design models to authoring tools, in: $A u$ thoring Tools for Advanced Technology Learning Environment, T. Murray, S. Blessing and S. Ainsworth, eds, Kluwer Academic Publishers, Dordrecht, 2003, pp. 377-409.

[7] M. Busch and N. Koch, Rich internet applications, state-of-the-art, Technical Report 0902, Programming and Software Engineering Unit (PST) Institute for Informatics, Ludwig-Maximilians-Universität München, Germany, 2009, available at: http://uwe.pst.ifi.lmu.de/publications/maewa_rias_report.pdf.

[8] K. Cagle, HTML5 Graphics with SVG \& CSS3, O'Reilly, Media, 2014.

[9] L. Castro, Creating pop-up footnotes in EPUB3 (and thus in iBooks), Pigs, Gourds, and Wikis (blog) (May 22, 2012), available at: http://www.pigsgourdsandwikis.com/2012/05/creating-pop-up-footnotes-in-epub-3-and.html.

[10] R. Darnton, in: The Case for Books: Past, Present, and Future, New York, 2009, p. 76.

[11] EPUB3 The standard, 2013, International Digital Publishing Forum (IDPF), available at: http://idpf.org/epub/30. 
[12] W. Grosso, Laszlo: An open source framework for, rich internet applications, 2005, available at: https://today.java.net/pub/ a/today/2005/03/22/laszlo.html.

[13] N.W. Jankowski, A. Scharnhorst, C. Tatum and Z. Tatum, Enhancing scholarly publications: Developing hybrid monographs in the humanities and social sciences, Scholarly and Research Communication 4(1) (2013), 1-26.

[14] E. Knutov et al., AH 12 years later: a comprehensive survey of adaptive hypermedia methods techniques, New Review of Hypermedia and Multimedia 15 (2009), 5-38.

[15] S. Woutersen-Windhouwer and R. Brandsma, Enhanced publications: state-of-the-art, DRIVER II report, 2009, available at: http://www.driver-repository.eu/component/option,com_jdownloads/Itemid,83/task,view.download/cid,53/. 